\title{
SIMBA: an Approach for Real-Time Multi-Agent Systems
}

\author{
V. Julian, C. Carrascosa, M. Rebollo, J. Soler and V. Botti \\ Dept. Sistemes Informàtics i Computació. \\ Universitat Politècnica de València. \\ \{vinglada,carrasco,mrebollo,jsoler,vbotti\}@dsic.upv.es
}

\begin{abstract}
The use of the agent/multi-agent system paradigm has increased sharply as an important field of research within the Artificial Intelligence area. In recent times, the application of this paradigm seems appropriate for solving complex problems which require intelligence and bounded response times. This paper presents SimBA : an architecture based on ARTIS agents as its main component for the development of real-time multi-agent systems. The ARTIS agent architecture guarantees an agent response that satisfies all its critical temporal restrictions in a real-time environment. The main feature of Simba systems is their applicability for complex, distributed, real-time domains. The architecture allows the communication among agents taking into account their hard temporal restrictions.

In order to show the use of systems of this kind, the paper describes the design of a multi-agent system for the distributed intelligent control of a residential building.
\end{abstract}

Keywords: agents, multi-agent systems, real-time AI.

\section{Introduction}

Over the last few years, the application of the agent / multi-agent paradigm in real-time environments arises from the required capacities of new real-time systems. This paradigm attempts to incorporate flexibility and distribution in new real-time designs. A Real-Time System (RTS) is a system in which the correctness of the system depends not only on the logical result of computation, but also on the time at which the results are produced [15]. It is well-known that a RTS is formed by a set of tasks characterised by a deadline, a period, a worst-case execution time and an assigned priority. A deadline defines the greatest time interval in which the system can provide a response. If the response is obtained after this time, it will probably not be useful. Researchers differentiate between two types of RTS. The first, called Hard Real-Time System, is a RTS where the execution of a task after its deadline is completely useless. Systems of this kind are critical systems and if timing responses are not satisfied, this will result in severe consequences. The second, called Soft Real-Time System, is characterised by the fact that the execution of a task after its deadline only decreases the quality of the task result [16]. Different techniques are needed for hard and soft RTS.

On the other hand, a multi-agent system which involves several agents that collaborate towards the achievement of a joint objective is viewed as a team of agents. Most proposed teamwork structures [10] [3] rely on agents in a multi-agent system to negotiate and/or contract with each other in order to initiate team plans. However, in dynamic, realtime domains with limited communication, complex negotiation protocols may take up too much time. Therefore, these protocols are not suitable for time-bounded problems.

Our work has been focused in time critical environments in which the full system can be controlled by autonomous agents that need communication to improve the system goal. This focus motivates the introduction of Social Real-Time Domains. In such domains, agents need to act autonomously while still working towards a common system goal. Time-critical environments require real-time response and, therefore, they eliminate the possibility of excessive communication among 
agents.

According to these concepts, it is possible to define a Real-Time Agent as an agent with temporal restrictions. These restrictions may be hard, soft or both. A real-time agent should guarantee its temporal restrictions and, concurrently, it should try to accomplish its goals. Finally, if a real-time agent is included as a component of a multi-agent system, this system can be considered as a Real-Time Multi-Agent System. It is important to highlight that the agent may have its interactions bounded. This modification will affect all the communication processes in the multi-agent system.

An architecture for a real-time multi-agent system is presented in this paper. The proposal is called Simba (Multi-Agent System Based on ARTIS ) and it constitutes a significant extension of the ARTIS agent architecture approach for real-time environments [2]. Simba can be seen as a set of ARTIS agents and their interactions. This proposal increases the applicability of the ARTIS agent architecture for problems where a multi-agent approach is more suitable than a centralised one.

The rest of the paper is structured as follows: section 2 focuses on the features of social real-time domains. Section 3 presents an overview of the SIMBA architecture for real-time environments and its main component, the ARTIS agent. Section 4 goes into the communicative aspects of this type of agents. In section 5 , an intelligent building management system is described as an example. Finally, some conclusions are mentioned in section 6 .

\section{Social Real-Time Domains}

We define a social real-time domain as a domain with the following characteristics:

- There is a team of autonomous agents $\mathcal{A}$ that collaborate to achieve a common long-term goal $\mathcal{G}$.

- Periodically, each agent can read or send messages $m$ with no adverse effects upon the achievement of $\mathcal{G}$.

- The domain is dynamic and time-bounded. This means that team performance is adversely affected if an agent fails to act for a period. Each agent is able to manage hard real-time restrictions and to solve a system subproblem.
- The domain has unreliable communication, either in terms of transmission reliability or bandwidth limits. If an agent $a_{i} \in \mathcal{A}$ sends a message $m$ to agent $a_{j} \in \mathcal{A}$, then $m$ arrives with some probability; or an agent $a_{i}$ can only receive $x$ messages every $y$ time units. That is, sending a message does not guarantee its reception.

- A message $m$ has a maximum length $K$, and an agent can send and receive at least one message $m$ with a period $p_{i}$.

In the extreme case, the communication channel lose all the messages, then an interval of no communication will appear, requiring the agents to act completely isolated. If agent $a_{i}$ cannot carry on with its action until receiving a message from $a_{j}$, then the team's performance could suffer. Because of the unreliable communication, the message might not get through on the first try. And because of the dynamic, real-time nature of the domain, the team's likelihood or efficiency of achieving $\mathcal{G}$ is reduced. However, if a message is received, it will be treated in a finite amount of time.

Intelligent building sensing and control is a social real-time domain since a set of agent controls different subsystems and they need to communicate with each other in order to maintain the global objective [13] [1]. There are several other examples of social real-time domains, such as port container terminal management, factory maintenance, distributed industrial process control and robot team control.

\section{SIMBA Architecture}

SimBA is an architecture for multi-agent systems to work properly in social real-time domains. The SimBA architecture constitutes the natural evolution of the ARTIS agent architecture, since it allows for the development of different related agents for hard real-time environments.

Basically, a SimbA system is formed by a set of ARTIS agents (AA) with probably hard temporal restrictions. This set of agents controls the subsystem of the real-time environment with hard critical constrains. Additionally, the system may integrate different types of agents, which cover other noncritical activities on the system. For this reason, Simba must be prepared to incorporate heterogeneous agents using standard agent-interaction processes. 
As mentioned above, the main component of the SIMBA architecture is the ARTIS agent, so, the architecture of this special type of agent is presented in the following point. Later, the formal approach and design aspects of the SIMBA architecture are described.

\subsection{The ARTIS Agent Architecture}

The ARTIS architecture is an extension of the blackboard model [12] which has been adapted to work in hard real-time environments. This architecture includes the use of well-known techniques of Real Time Artificial Intelligence Systems [9]. This approach guarantees reacting on the environment in a dynamic and flexible way. It incorporates all the necessary aspects that the agency features provide to a software system, but adapted to hard real-time environments.

The ARTIS agent architecture guarantees an agent response that satisfies all the critical temporal restrictions of the system, its capacities for problem-solving, for adaptability and for proactivity help to provide the best answer for the current environment status. Its critical timing requirements are $100 \%$ guaranteed by means of an off-line schedulability analysis as detailed in [8].

Basically, the agent must perceive an environment through a set of sensors. After this, the system must compute and transmit a response to other agents or the environment using a set of effectors. The response can be obtained after a reflex process or a deliberative process. Furthermore, the agent must work with hard temporal restrictions in dynamic environments.

Though the basic AA is designed to work properly under hard real-time restrictions, some of the optional features (such as communication with other agents) may prevent this real-time behaviour due to the unpredictable actions they involve. Therefore, it is the agent designer's decision to choose which features (and, therefore, which behaviours) the agent is going to have.

The AA architecture could be labeled as a vertical-layered, hybrid architecture with added extensions to work in a hard real-time environment [11]. It is formed by the following elements (see Figure 1):

- A set of sensors and effectors to be able to interact with the environment. Perception and action processes are time-bounded. The com- munication acts are included into these processes. So, the ArTis agent has a Communication Module (CoMo) which is in charge of controlling the sending and arrival of messages.

- A set of in-agents (internal agents) that models the AA behaviours in order to achieve the AA goals. An in-agent is an internal entity that has the necessary knowledge to solve a particular problem (this knowledge can incorporate IA techniques that provide intelligence for solving the problem). Basically, this entity periodically performs a specific task (which may or may not be complex). The main reason for splitting the whole problem-solving method into smaller entities is to provide an abstraction which organises the problemsolving knowledge in a modular and gradual way. Depending on the temporal restrictions and the intelligence used in its problem-solving method, in-agents can be classified as critical or acritical. A critical in-agent is characterised by a period and a deadline. The available time for the in-agent to obtain a valid response is bounded. It must guarantee a basic response to the current environment situation. It is formed by two layers (see Figure 1): the reflex layer and the real-time deliberative layer. The first one assures a minimal quality response and the second one tries to improve this response. The reflex layer of all the in-agents make up the AA mandatory phase. On the other hand, the real-time deliberative layers form the optional phase. An acritical in-agent only has the real-time deliberative layer. Almost all the in-agents for real-time environments are critical in-agents.

- A set of beliefs comprising a world model (with all the domain knowledge which is relevant to the agent) and the internal state. This set is stored in a frame-based blackboard. Temporal extensions have been incorporated allowing the reasoning entities to manage time naturally.

- The Control Module that is responsible for the real-time execution of the in-agents that belong to the AA. The temporal requirements of the two in-agent layers (reflex and deliberative) are different. Thus, the control module must employ different execution criteria for each one. The control module of an AA is divided into 
two submodules: the reflex server and the deliberative server.

- Reflex server (RS) This module is in charge of controlling the execution of reactive components, that is, the components with critical temporal restrictions. Due to these restrictions, it is integrated within a Real-Time Operating System (RTOS).

- Deliberative server (DS) This module is in charge of controlling the execution of deliberative components. Therefore, this server is the intelligent element of the control module, but with temporal restrictions.

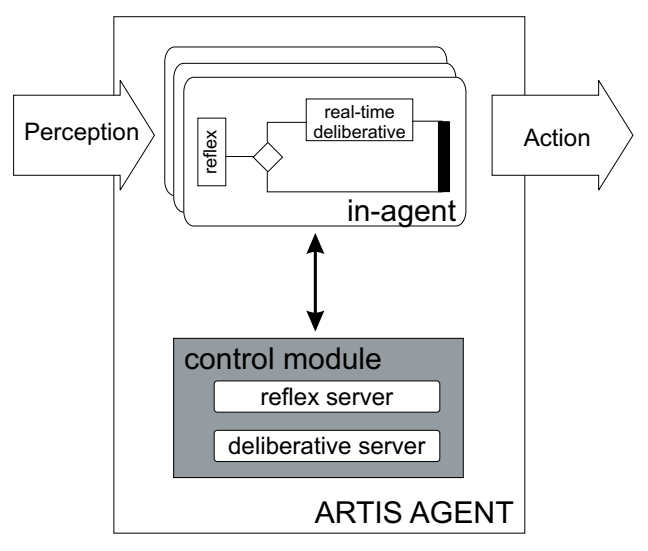

Figure 1: ARTis Agent Architecture

\subsection{Formal Approach}

Once the ARTIS agent architecture is presented, a formal view of the SIMBA approach is described. This formal view allows us to explain the architecture in a more concrete way.

Definition $1 A$ SIMBA architecture is an structure:

$$
\text { SimBA }=\langle A, O, L, I\rangle
$$

where:

- $A=\left\{A A_{i}\right\}$, a countable set of ARTIS agents, which are formalised below.

- $O=$ a domain ontology, which specifies the common vocabulary in order to represent the system environment.
- $L=$ a communication language to be employed by the agents for the interaction processes.

- $I=\left\{\left(A A_{i}, A A_{j}\right) / A A_{i}, A A j \in A\right\}$, a set of interactions represented by $A A$ pairs. These interactions show the relations in the system organisation.

As can be seen, the main component of a SimBA system is the ARTIS agent, which can be formally defined as follows:

Definition 2 An ARTIS agent is an structure:

$$
A A_{i}=\left\langle\operatorname{Behav}_{i}, G_{i}, B_{i}, \beta_{i}\right\rangle
$$

where:

- Behavi is a set of different behaviours of the $A A_{i}$ for different situations.

- $G_{i}=\left\{g_{1}^{\left[t_{1}, t_{2}\right]}, g_{2}^{\left[t_{2}, t_{3}\right]}, \ldots, g_{n}^{\left[t_{n}, t_{m}\right]}\right\}$, a set of $A A$ goals, representing the motivations and restrictions of the agent. The goals are designed as desired states and they can be temporal bounded. These temporal restrictions show the interval in which the goal must be obtained. The goals can be expressed as $\mathrm{RTCTL}^{*}$ formulae [5].

- $B_{i}$ is the $A A_{i}$ belief set in the current instant. All the data in this set are time stamped using a temporal logic as explained in [4].

- $\beta_{i}: G_{i} \times B_{i} \rightarrow$ Behav $_{i}$, a selection function that determines the current behaviour of the $A A_{i}$ according to its goals and its beliefs. This function corresponds to the AA Control Module.

Each behaviour is defined as follows

Definition 3 A behaviour beh $\in$ Behav $_{i}$ is a set of in-agents

$$
b e h=\left\{a_{i j}\right\}
$$

A behaviour contains the problem-solving knowledge. As stated above, an in-agent gives a solution for a particular problem of the ARTIS agent.

Definition 4 An in-agent is an structure

$$
a_{i j}=\left\langle\rho_{i j}, f \rho_{s e l}, \sigma_{i j}, f \sigma_{s e l}, B_{i j}, D_{i j}, T_{i j}\right\rangle
$$

where: 
- $\rho_{i j}$ is a set of reflex actions that can be executed by the in-agent.

- $f \rho_{\text {sel }}: B_{i j} \times\left(D_{i j}, T_{i j}\right) \rightarrow \rho_{i j}$, a selection function that determines the appropriate reflex action to execute by the in-agent according to its current state and temporal restrictions.

- $\sigma_{i j}$ is a set of cognitive actions that can be executed by the in-agent.

- $f \sigma_{\text {sel }}: B_{i j} \times\left(D_{i j}, T_{i j}\right) \rightarrow \sigma_{i j}$, a selection function that determines the appropriate cognitive action to execute by the in-agent according to its current state and temporal restrictions.

- $B_{i j} \subset B_{i}$, is a set of beliefs representing the internal state and environment of the in-agent. These beliefs are part of the belief set $\left(B_{i}\right)$ of the $A A_{i}$ that the in-agent belongs to.

- $D_{i j}$ is a deadline, which indicates the greatest time interval in which the in-agent should have executed an action.

- $T_{i j}$ is the period, which determines the activation frequency of the in-agent. This period is needed due to the external environment's own dynamic of RTS. At each period, the in-agent execution begins reading the new incoming values of the data (perceptions) through the appropriate sensors. These new values update the $B_{i j}$ of the in-agent.

This organisation among AA, behaviours and inagents provides a hierarchy of abstractions which structures the problem-solving knowledge in a modular and gradual way. One of the main reasons for doing this is to employ the advantages of modular programming, that is, complexity split and code reusability.

\subsection{Architecture Design}

The SimBA architecture design is FIPA-compliant in order to allow for future inter-communication with outer agents from other platforms. A FIPA agent platform is defined as software that implements the set of FIPA specifications. To be considered FIPA-compliant, an agent platform implementation must at least implement the Agent Management [7] and Agent Communication Language specifications [6]:

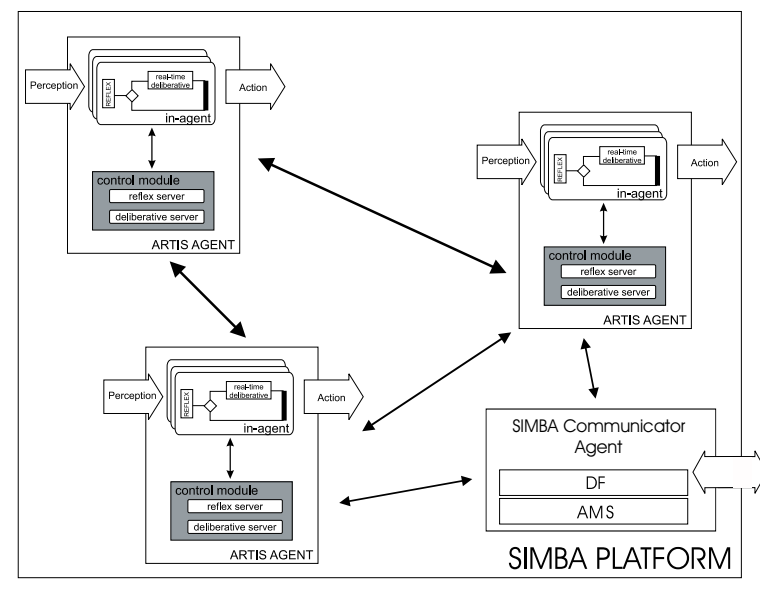

Figure 2: SimBA Platform

- The Agent Management Specification forces the implementation of a Directory Facilitator (DF) that provides yellow-pages service to the agents involved and an Agent Management System (AMS) which maintains the addresses for agents registered in the platform (whitepages service).

- The Agent Communication Language Specification contains the message description set to be employed in agent interactions.

A graphical view of the SIMBA architecture is shown in Figure 2 in accordance with these considerations. As can be seen, it is formed by several ARTIS agents and a mediator agent which integrates the DF and the AMS services. This mediator agent is the SImBA interface with agents that do not follow the ARTIS agent architecture. Moreover, the communication language in SIMBA will obviously be FIPA-ACL.

With respect to each ARTIS agent design, a single AA is implemented through a graphical toolkit named InSiDE (Integrated Simulation and Development Environment) [14] which facilitates the design and debugging of an AA. InSiDE is a visual toolkit which was developed to allow for agentoriented implementation and management of ARTIS agents. It incorporates an off-line schedulability analysis, which assures that all the temporal restrictions are guaranteed a priori. By means of this toolkit, a user can build a prototype of an ARTIS Agent, which is directly executable over the RTLinux operating system [8]. 


\section{ARTIS Agent communica- tive aspects}

The communication process has to be timebounded in some way in order to be considered within a real-time restricted system such as the AA. On the other hand, the whole execution time of this process cannot be bounded, due to the multiple factors that may affect it, making it very difficult to assure the sending and reception of answers. Moreover, the foreseen interaction processes may be complex, and they may need to send several messages in the same process.

The main use of the AA communication is to ask for a service. According to its possible uses, it doesn't seem appropriate to consider the unfulfillment of a communication process as catastrophic. So, even though the communication process has soft real-time restrictions, it is not possible to assign critical temporal restrictions to manage it.

As has been previously mentioned, the part of the Control Module in charge of controlling the execution of the non-critical tasks of the AA is the Deliberative Server (DS). The DS controls the execution of the Communication Module. This module is in charge of controlling all the low-level peculiarities of a communication process such as message construction (according to the communication language), physical media sending and receiving, parsing, syntactic checking, etc.

Figure 3 shows a possible execution stage of an AA by a timing diagram. In this example, the AA is comprised by two in-agents ( $a$ and $b$ ). Black boxes represent the processor time intervals assigned to the in-agent reflex layer execution. Between these executions, there exists available time (white boxes). At the beginning of each one of these slacks, the DS is executed and it schedules this time taking into account the execution of the Communication Module and the real-time deliberative layers of the in-agents. The DS only launches the Communication Module if there is enough slack time to assure the sending of at least one message and the reception of at least another message.

This way of controlling the communication makes it possible to communicate without interfering in the fulfillment of critical AA restrictions.

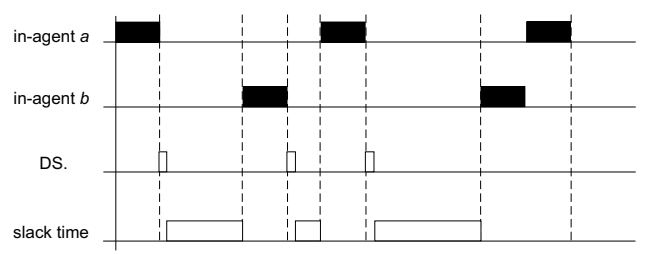

Figure 3: Timing diagram example

\section{Example: Intelligent Build- ings}

In this paper, we present a prototype of a system, developed according to our approach, which emphasises the multi-agent architecture presented in previous sections. The system we present controls an intelligent building. An intelligent building is one that utilises computer technology to autonomously govern the building environment so as to optimise user comfort, energy-consumption, safety and monitoring-functions [13]. In this example, we consider a residential building that has distributed different sensor and actuator devices which can be controlled and monitored. Due to the limits of the paper only some aspects of the example are sketched.

Three main functionalities can be extracted from the given definition of an intelligent building:

- Safety \& Security: the system must take into account system failures such as gas or water leaks and detection of intruders.

- Comfort: all the aspects related to the building habitability and resident preferences.

- Energy-consumption: related to an effective management of energy resources such as electricity, gas, diesel or water.

This system belongs to the social real-time domains explained in section 2. There exist critical temporal constraints such as alarm activation or closing/opening valves. Moreover, there exists a clear distribution in the system activities. The coordination among the entities in the system will improve the global functionality but is not critical for the fulfillment of the critical constraints.

In this case, the SIMBA system is formed by three agents (see Figure 4): the safety/security agent, the comfort agent and the energy agent. Each one is in charge of controlling its corresponding subsystem. 


$$
A=\left\{A A_{\text {safety }}, A A_{\text {comfort }}, A A_{\text {energy }}\right\}
$$

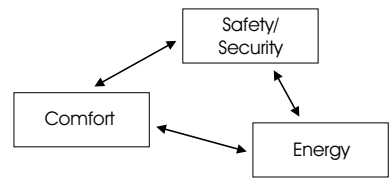

Figure 4: Intelligent building control system

Each one of these agents is an ARTIS agent. SImBA allows for the interaction with external, nonARTIs agents through the services supplied by the mediator agent. For instance, it is possible for an agent to ask the system about one of the residents. The system can verify whether this person is at home or not. Some interactions in the system are:

- The safety/security agent informs about a system failure to the rest of the agents.

- The safety/security agent informs the comfort agent that the building is empty in order to minimise its functionalities.

- The energy agent and the comfort agent negotiate different energy resources consumption.

Due to the limits of this paper, we will focus on the safety/security agent. Depending on the presence or absence of residents in the building, the agent shows two different behaviours. If there is nobody in the building, then this agent is the only entity responsible for safety and security. The difference between them lies in the control that the agent must exert on the building. When it is unoccupied, the safety/security agent assumes all the responsibilities.

Additionally, another case can be detected: when a failure has happened-leak or intrusion-and the agent must change its monitoring behaviour to another, more critical and reactive, to handle it. Figure 5 shows the different behaviours of the safety/security agent as an state diagram.

$$
\text { Behav }_{\text {safety }}=\left\{b e h_{o c c}, \text { beh } h_{\text {empty }}, \text { beh } h_{\text {emerg }}\right\}
$$

Each behaviour must deal with two responsibilities - leaks and intruders-. So, it seems adequate to assign an in-agent to each one. In such a case, the in-agents for each behavior only vary their temporal parameters - deadline

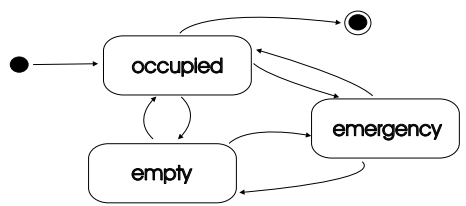

Figure 5: State diagram. Safety agent behaviours

and period-. Their knowledge and their reactions must be the same ones.

$$
\text { beh } h_{\text {empty }}=\left\{a_{\text {leak }}, a_{\text {secur }}\right\}
$$

Temporal characteristics of in-agents will take into account the differences in the sensors and actuators sampling. One usual security measure in classic control systems is to achieve that the period of the system is $\frac{T}{2}$, where $\mathrm{T}$ is the period of the highest-frequency device. The deadline estimation depends on the dynamic of the environment. It must be such a deadline that allows to solve the problem in a satisfactory way for the user. For instance, to raise the fire alarm 2 seconds later from to detect smoke. It always must be lower than the period.

$$
a_{\text {leak }}=\left\{\rho_{\text {leak }}, 5,2\right\} \quad a_{\text {secur }}=\left\{\rho_{\text {secur }}, 30,10\right\}
$$

It must be always guarantee that the system can achieve its goals. That's why temporal intervals which characterise the goals must be less restrictive than the deadline and period of the in-agent responsible of the goal.

$$
\square G\left(\text { smoke }=\text { TRUE } \rightarrow \square F^{\leq 10} \text { alarm }=O N\right)
$$

In this RTCTL* expression, we are asking the system for a deadline of 10 seconds from the detection of smoke to the alarm raising.

\section{Conclusions}

This paper shows the SIMBA architecture, which is a real-time multi-agent system architecture. This architecture shows how a multi-agent oriented paradigm can be applied to solve problems in social real-time domains. To do so, it is needed software architecture with the appropriate components along with development artifacts adapted to build this kind of systems.

The work described in this paper is the result of an investigation effort with respect to the application of interaction processes in an ARTIS agent. 
Several questions remain open with respect to new protocols for ARTIS agents in order to do complex cooperation or coordination processes.

We are currently working on the implementation of a complete version of the example described in this paper. This prototype will allow us to analyse the influence of the communication processes within the real-time behaviour and to prove new interaction protocols.

\section{References}

[1] M. Boman, P. Davidsson, and H. Younes. Artificial decision making under uncertainty in intelligent buildings. In Proceedings of UAI'99, 1999.

[2] V. Botti, C. Carrascosa, V. Julian, and J. Soler. Modelling agents in hard real-time environments. In MAAMAW'99 Proceedings, volume 1647 of $L N A I$, pages 63-76. SpringerVerlag, 1999.

[3] P. Cohen, H. Levesque, and I. Smith. On team formation. In Contemporary Action Theory. Synthese. J. Hintikka and R. Tuomela, editors, 1998.

[4] A. Crespo, V. Botti, F. Barber, D. Gallardo, and E. Onaindia. A temporal blackboard for real-time process control. Engineering Applications of Artificial Intelligence, pages 225-256.

[5] E. Emerson, A. Mok, A. Sistla, and J. Srinivasan. Quantitative temporal reasoning. RealTime Systems, (4):331-352, 1992.

[6] FIPA. Agent acl message structure specification. Technical Report XC00061E, 2001.

[7] FIPA. Agent management specification. Technical Report XC00023H, 2001.

[8] A. Garcia-Fornes, A. Terrasa, V. Botti, and A. Crespo. Analyzing the schedulability of hard real-time artificial intelligence systems. Engineering Applications of Artificial Intelligence, pages 369-377, 1997.

[9] A. Garvey and V. Lesser. A survey of research in deliberative real-time artificial intelligence. The Journal of Real-Time Systems, (6):317347, 1994.
[10] B. J. Grosz. Collaborative systems. AI Magazine, 17(2):67-85, 1996.

[11] J. P. Muller. A conceptual model for agent interaction. In Proceedings of the second International Working Conference on Cooperating Knowledge Base Systems, pages 213-234, DAKE Centre, University of Keel, 1994. Deen, S. M. (Ed.).

[12] P. Nii. Blackboard systems: The blackboard model of problem solving and the evolution of blackboard architectures. The AI Magazine, pages 38-53, Summer 1986.

[13] S. Sharples, V. Callaghan, and G. Clarke. A multi-agent architecture for intelligent building sensig and control. Sensor Review, 19(2):135-140, 1999.

[14] J. Soler, V. Julian, C. Carrascosa, and V. Botti. Applying the artis agent architecture to mobile robot control. In Proceedings of IBERAMIA'2000. Atibaia, Sao Paulo, Brasil, volume I, pages 359- 368. Springer Verlag, 2000 .

[15] J. Stankovic. Misconceptions about real-time computing. IEEE Computer, 12(10):10-19, 1988.

[16] J. Stankovic. Distributed real-time computing: The next generation. Journal of the Society of Instrument and Control Engineers of Japan, 1992. 